\title{
ЧАСТОТА РЕЦИДИВОВ ПОСЛЕ РАДИОЙОДТЕРАПИИ ПРИ ДИФФУЗНОМ ТОКСИЧЕСКОМ ЗОБЕ У ДЕТЕЙ В ЗАВИСИМОСТИ ОТ ОБЪЕМА ЩИТОВИДНОЙ ЖЕЛЕЗЫ
}

\author{
Иванникова Т.E., Нагаева Е.В. \\ ФГБУ «НМИЦ эндокринологии» Минздрава России, Москва
}

Диффузный токсический зоб (ДТЗ) - самая частота причина тиреотоксикоза у детей, ежегодно в Российской Федерации диагностируется 600-800 новых случаев. Современные методы лечения Дт3 включают тиреостатическую терапию, хирургическое лечение и радиойодтерапию. Радиойодтерапия (РЙТ) является наиболее молодым и активно развивающимся в последнее время методом радикального лечения ДТЗ в детском возрасте.

ЦЕЛЬ: исследования - оценка влияния исходного объема щитовидной железы на частоту рецидивов после радиойодтерапии.

МАТЕРИАЛЫ И МЕТОДЫ: Ретроспективное исследование результатов лечения диффУзного токсического зоба у 43 детей и подростков (39 девочек, 90.7\%), получивших РЙТ (активности 500-1300 мБк) в период с января 2016г по сентябрь 2019г в ФГБУ «НМИЦ эндокринологии» Минздрава России. Перед проведением РЙТ с целью индивидуального подбора дозы радиофармпрепарата всем пациентам проводилось индивидуальное дозиметрическое планирование.

РЕЗУЛЬТАТЫ: на момент проведения РЙТ хронологический возраст (Ме [25; 75]) пациентов составил

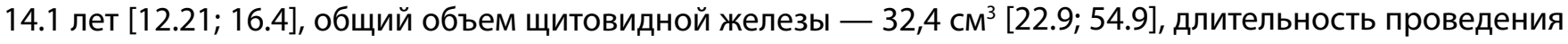
тиреостатической терапии до применения РЙТ - 3.54 года $[2.42 ; 5.75]$, период наблюдения после проведения РЙТ - 1,84 лет $[1.21 ; 2.77]$.

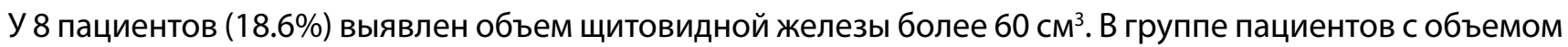
щитовидной железы менее $60 \mathrm{~cm}^{3}$ за весь период наблюдения рецидивов ДТ3 не наблюдалось. Среди пациентов, у которых объем щитовидной железы превысил $60 \mathrm{~cm}^{3}$, несмотря на индивидуальное дозирование радиофармпрепарата, в 37.5\% случаев $(\mathrm{N}=3)$ диагностирован рецидив тиреотоксикоза, потребовавший повторного курса РЙТ.

ВЫВОдЫ: полученные данные об эффективности РЙТ при лечении ДТЗ у детей и подростков свидетельствуют о возможной связи между объемом щитовидной железы и риском рецидива заболевания. Данные требуют подтверждения на выборках с большей численностью пациентов. 